\title{
Phase Reconstruction of Strong-Field Excited Systems by Transient-Absorption Spectroscopy
}

\author{
Zuoye Liu, ${ }^{1,2, *}$ Stefano M. Cavaletto, ${ }^{1, \dagger}$ Christian Ott, ${ }^{1}$ Kristina Meyer, ${ }^{1}$ Yonghao Mi, ${ }^{1}$ Zoltán Harman, ${ }^{1}$ \\ Christoph H. Keitel, ${ }^{1}$ and Thomas Pfeifer, ${ }^{1, \$}$ \\ ${ }^{1}$ Max-Planck-Institut für Kernphysik, Saupfercheckweg 1, 69117 Heidelberg, Germany \\ ${ }^{2}$ School of Nuclear Science and Technology, Lanzhou University, 730000 Lanzhou, China
} (Received 1 April 2015; published 13 July 2015)

\begin{abstract}
The evolution of a $V$-type three-level system is studied, whose two resonances are coherently excited and coupled by two ultrashort laser pump and probe pulses, separated by a varying time delay. We relate the quantum dynamics of the excited multilevel system to the absorption spectrum of the transmitted probe pulse. In particular, by analyzing the quantum evolution of the system, we interpret how atomic phases are differently encoded in the time-delay-dependent spectral absorption profiles when the pump pulse either precedes or follows the probe pulse. This scheme is experimentally applied to atomic Rb, whose finestructure-split $5 s^{2} S_{1 / 2} \rightarrow 5 p^{2} P_{1 / 2}$ and $5 s^{2} S_{1 / 2} \rightarrow 5 p{ }^{2} P_{3 / 2}$ transitions are driven by the combined action of a pump pulse of variable intensity and a delayed probe pulse. The provided understanding of the relationship between quantum phases and absorption spectra represents an important step towards full timedependent phase reconstruction (quantum holography) of bound-state wave packets in strong-field lightmatter interactions with atoms, molecules, and solids.
\end{abstract}

DOI: 10.1103/PhysRevLett.115.033003

PACS numbers: 32.80.Qk, 32.80.Wr, 42.65.Re

A long-standing dream in the physics of light-matter interaction is the observation of the quantum dynamics of electrons bound in atoms and molecules [1-3]. Traditional spectroscopy methods enable one to access the evolution of the populations, i.e., the probability that a quantum system is in a given state. Much more demanding is the extraction of the associated quantum phases.

With the development of femtosecond and attosecond laser pulses, transient-absorption spectroscopy (TAS) has emerged as a powerful method to gain amplitude and phase information on the evolution of a quantum system [4-9]. In such pump-probe geometry [Fig. 1(a)], a short pump pulse of tunable intensity excites a quantum system, whose ensuing dynamical evolution is measured by a weak probe pulse. The absorption spectrum of the transmitted field results from the interference of the incoming probe pulse with the electric field emitted by the system [10]. Since this interference pattern depends on the state of the system at the arrival of the probe pulse, its quantum-mechanical evolution can be time resolved by modifying the interpulse delay. In contrast to most strong-field studies relying on the ionization of the system [11,12], this enables direct access to bound-state dynamics.

Very recently, experiments started to investigate the absorption spectrum of a weak probe pulse which excites a coherent superposition of quantum states and is followed by a strong laser field $[7,13,14]$. For nonautoionizing bound states, in the absence of this subsequent pulse, well-known symmetric Lorentzian line shapes are observed. However, an additional strong pulse can be employed to nonlinearly drive the state generated by the probe field, manipulating the absorption lines into, e.g., asymmetric Fano-like shapes [15-17]. Although this opens further prospects for line-shape quantum control $[18,19]$, it still remains an open question how phase information is encoded in the time-delay-dependent strong-field absorption spectrum.

Here, we investigate theoretically and experimentally the phase information which can be extracted from transientabsorption spectra for both above-mentioned scenarios, i.e., when the dynamics are either triggered or modified by an intense pump pulse. The $V$-type three-level scheme depicted in Fig. 1(b) is used to describe an ensemble of $\mathrm{Rb}$ atoms, with fine-structure-split excited states. From the

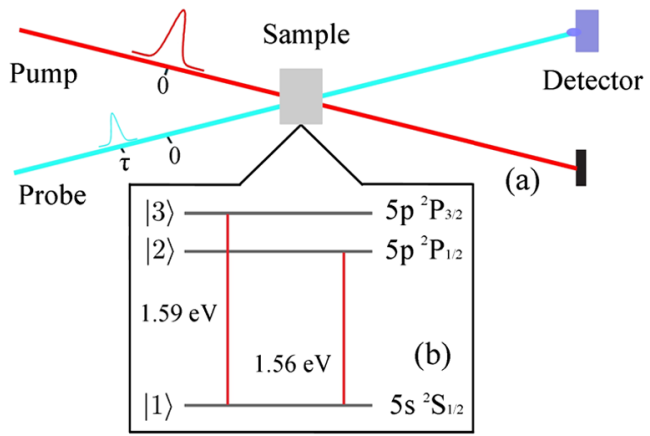

FIG. 1 (color online). (a) Transient-absorption-spectroscopy setup in which, upon interaction of an atomic ensemble with two pulses separated by a time delay $\tau$, the absorption spectrum of the transmitted probe pulse is measured. (b) $V$-type three-level scheme used to model the ensemble of $\mathrm{Rb}$ atoms interacting with the laser pulses. 
ground state, the single-electron transitions $5 s^{2} S_{1 / 2} \rightarrow$ $5 p^{2} P_{1 / 2} \quad(|1\rangle \rightarrow|2\rangle$ at $794.76 \mathrm{~nm})$ and $5 s^{2} S_{1 / 2} \rightarrow$ $5 p^{2} P_{3 / 2}(|1\rangle \rightarrow|3\rangle$ at $780.03 \mathrm{~nm})$ are excited by fsduration, pump or probe optical pulses centered at $780 \mathrm{~nm}$ and separated by a time delay $\tau$. We focus on the oscillations in $\tau$ exhibited by the absorption spectrum of a weak probe pulse [20] and show how these oscillating features differently encode, for positive and negative time delays, the dependence of the atomic phases upon pump-pulse intensities.

The atomic system is described by the density matrix $\hat{\rho}(t, \tau)$ [21], of elements $\rho_{i j}(t, \tau), i, j \in\{1,2,3\}$. An offdiagonal element $\rho_{i j}(t, \tau), i \neq j$, represents the coherence between states $|i\rangle$ and $|j\rangle$, while a diagonal element $\rho_{i i}(t, \tau)$ quantifies the population of level $|i\rangle$ at energy $\omega_{i}$, with transition energies $\omega_{i j}=\omega_{i}-\omega_{j}$ given by $\omega_{21}=1.56 \mathrm{eV}$ and $\omega_{31}=1.59 \mathrm{eV}$. Here, atomic units are used unless otherwise stated. The probe pulse is centered at $t=\tau$ and is modeled by the classical field $\mathcal{E}_{\mathrm{pr}}(t, \tau)=$ $\mathcal{E}_{\mathrm{pr}, 0} f(t-\tau) \cos \left[\omega_{L}(t-\tau)+\phi_{\mathrm{pr}}\right] \hat{\boldsymbol{e}}_{z}$, with the unit polarization vector $\hat{\boldsymbol{e}}_{z}$ along the $z$ direction, the laser frequency $\omega_{L}=1.59 \mathrm{eV}$, the initial phase $\phi_{\mathrm{pr}}=0$, and the peak field strength $\mathcal{E}_{\mathrm{pr}, 0}=\sqrt{8 \pi \alpha I_{\mathrm{pr}}}$ related to the peak intensity $I_{\mathrm{pr}}$ and the fine-structure constant $\alpha$. The envelope function is modeled by $f(t)=\cos ^{2}(\pi t / T) R(t / T)$, where $R(x)=$ $\theta(x+1 / 2)-\theta(x-1 / 2)$ is defined in terms of the Heaviside step function $\theta(x)$. Consequently, $f(t)$ is nonvanishing in an interval of duration $T=\pi T_{\mathrm{FWHM}} /$ [ $2 \arccos (\sqrt[4]{1 / 2})]$, with $T_{\mathrm{FWHM}}=30 \mathrm{fs}$ being the full width at half maximum (FWHM) of $f^{2}(t)$. Similarly, the pump pulse is modeled by $\mathcal{E}_{\mathrm{pu}}(t)=\mathcal{E}_{\mathrm{pu}, 0} f(t) \cos \left(\omega_{L} t+\right.$ $\left.\phi_{\mathrm{pu}}\right) \hat{\boldsymbol{e}}_{z}$, with initial phase $\phi_{\mathrm{pu}}=0$, peak field strength $\mathcal{E}_{\mathrm{pu}, 0}$, and peak intensity $I_{\mathrm{pu}}$. The pump pulse is centered at $t=0$, such that it precedes the probe pulse for $\tau>0$ (pumpprobe) and follows it for $\tau<0$ (probe-pump). Both linearly polarized pulses couple the ground state $|1\rangle \equiv 5 s^{2} S_{1 / 2}$, with magnetic quantum number $M= \pm 1 / 2$, to the two closely lying states $|2\rangle \equiv 5 p^{2} P_{1 / 2}$ and $|3\rangle \equiv 5 p^{2} P_{3 / 2}$, also with $M= \pm 1 / 2$ [22]. For the pulse intensities used, only electric-dipole-(E1-)allowed transitions with $\Delta M=0$ are considered, for which the dipole-moment matrix elements are $\boldsymbol{d}_{1 k}=d_{1 k} \hat{\boldsymbol{e}}_{z}, k \in\{2,3\}$, with $d_{1 k}$ well approximated by their nonrelativistic values, $d_{13}=d_{12} \sqrt{2}$ [20,23]. This allows us to introduce the two complex, time-dependent Rabi frequencies $\Omega_{R k}(t, \tau)=d_{1 k}\left[\mathcal{E}_{\mathrm{pr}, 0} f(t-\tau) e^{-i \omega_{L} \tau} e^{i \phi_{\mathrm{pr}}}+\right.$ $\left.\mathcal{E}_{\mathrm{pu}, 0} f(t) e^{i \phi_{\mathrm{pu}}}\right], \quad$ such that $\boldsymbol{d}_{1 k} \cdot\left[\mathcal{E}_{\mathrm{pu}}(t)+\mathcal{E}_{\mathrm{pr}}(t, \tau)\right]=$ $\left[\Omega_{R k}(t, \tau) e^{i \omega_{L} t}+\Omega_{R k}^{*}(t, \tau) e^{-i \omega_{L} t}\right] / 2$.

The atomic system is initially in its ground state, i.e., $\rho_{i j, 0}=\delta_{i 1} \delta_{j 1}$, with the Kronecker symbol $\delta_{i i^{\prime}}$. Its dynamical evolution results from the Liouville-von Neumann equation $\quad d \hat{\rho} / d t=-i\left[\hat{H}_{0}+\hat{H}_{\text {int }}(t, \tau), \hat{\rho}(t, \tau)\right]-\frac{1}{2}\{\hat{\Gamma}, \hat{\rho}(t, \tau)\}$, with $[\hat{A}, \hat{B}]=\hat{A} \hat{B}-\hat{B} \hat{A}$ and $\{\hat{A}, \hat{B}\}=\hat{A} \hat{B}+\hat{B} \hat{A} \quad$ [21]. Here, $\quad \hat{H}_{0}=\sum_{i=1}^{3} \omega_{i} \hat{\sigma}_{i i}$ is the electronic-structure
Hamiltonian, with ladder operators $\hat{\sigma}_{i j}=|i\rangle\langle j|$, while $\hat{H}_{\text {int }}(t, \tau)=-\frac{1}{2} \sum_{k=2}^{3} \Omega_{R k}(t, \tau) \hat{\sigma}_{1 k} e^{i \omega_{L} t}+$ H.c. is the $E 1$ light-matter-interaction Hamiltonian in the rotating-wave approximation. The diagonal matrix $\hat{\Gamma}$ has elements $\Gamma_{i j}=\delta_{i j} \gamma_{j}$, with $\gamma_{1}=0$ and $\gamma_{2}=\gamma_{3}=1 /(500 \mathrm{fs})$. These decay rates are chosen to approximate the experimental linewidths of $8 \mathrm{meV}$, much larger than the spontaneous decay rates. They effectively account for Doppler broadening and collision-induced broadening, as well as additional broadening in the nanosecond pedestal of the femtosecond pulses. For fs-duration pulses, these decay times are much longer than the interaction times and intensity-dependent phase effects are not significantly sensitive to $\gamma_{k}$.

The measured optical-density (OD) absorption spectrum is given by $\mathcal{S}(\omega, \tau)=-\log \left[S_{\mathrm{pr}, \text { out }}(\omega, \tau) / S_{\mathrm{pr} \text {,in }}(\omega)\right]$, where $S_{\mathrm{pr}, \text { in }}(\omega)$ and $S_{\mathrm{pr}, \text { out }}(\omega, \tau)$ are the spectral intensities of the incoming and transmitted probe pulse, respectively. The absorption spectrum is related to the single-particle dipole response of the system via [10]

$$
\mathcal{S}_{1}(\omega, \tau) \propto-\omega \mathbf{I m}\left[\sum_{k=2}^{3} \hat{\boldsymbol{e}}_{z} \cdot \boldsymbol{d}_{1 k}^{*} \int_{-\infty}^{\infty} \rho_{1 k}(t, \tau) e^{-i \omega(t-\tau)} d t\right],
$$

with Fourier transforms centered at the arrival time of the probe pulse. In the presence of a single pulse, the system, excited at $t=t_{0}$ into the state $\hat{\rho}\left(t_{0}\right)$, decays with time-dependent coherences $\rho_{1 k}(t)=$ $\rho_{1 k}\left(t_{0}\right) e^{i \omega_{k 1}\left(t-t_{0}\right)} e^{-\left(\gamma_{k} / 2\right)\left(t-t_{0}\right)}$, giving rise [Eq. (1)] to stationary lines centered on $\omega_{21}$ and $\omega_{31}$, with linewidths given by $\gamma_{2}$ and $\gamma_{3}$, respectively. However, if two pulses are used, the coherences $\rho_{1 k}(t, \tau)$ become explicitly time-delay dependent, implying a modulation in $\tau$ of intensity and shape of the spectral lines. Although rapid oscillations in $\tau$ characterize the absorption spectrum in Eq. (1), these fast oscillating features are averaged out for the noncollinear experimental geometry in Fig. 1(a). This is included in our model via convolution of the spectrum in Eq. (1) with a normalized Gaussian function $G(\tau, \Delta \tau)$ of width $\Delta \tau=5 \times 2 \pi / \omega_{L}$, thus obtaining

$$
\mathcal{S}(\omega, \tau)=\left\langle\mathcal{S}_{1}(\omega, \tau)\right\rangle_{\tau}=\int_{-\infty}^{\infty} G\left(\tau-\tau^{\prime}, \Delta \tau\right) \mathcal{S}_{1}\left(\omega, \tau^{\prime}\right) d \tau^{\prime} .
$$

In order to investigate the intensity-dependent phase information encoded in $\mathcal{S}(\omega, \tau)$, in Fig. 2 we compare numerical calculations of Eq. (2) for a probe intensity of $9.2 \times 10^{8} \mathrm{~W} / \mathrm{cm}^{2}$. This corresponds to pulse areas $\int\left|\Omega_{R k}(t, \tau)\right| d t=d_{1 k} \mathcal{E}_{\mathrm{pr}, 0} \int f(t) d t \approx 0.2 \pi$, preventing the generation of appreciable atomic phase differences. As shown in Fig. 2(a) for a pump intensity of $1.0 \times 10^{10} \mathrm{~W} / \mathrm{cm}^{2}$, the spectrum consists of two lines 


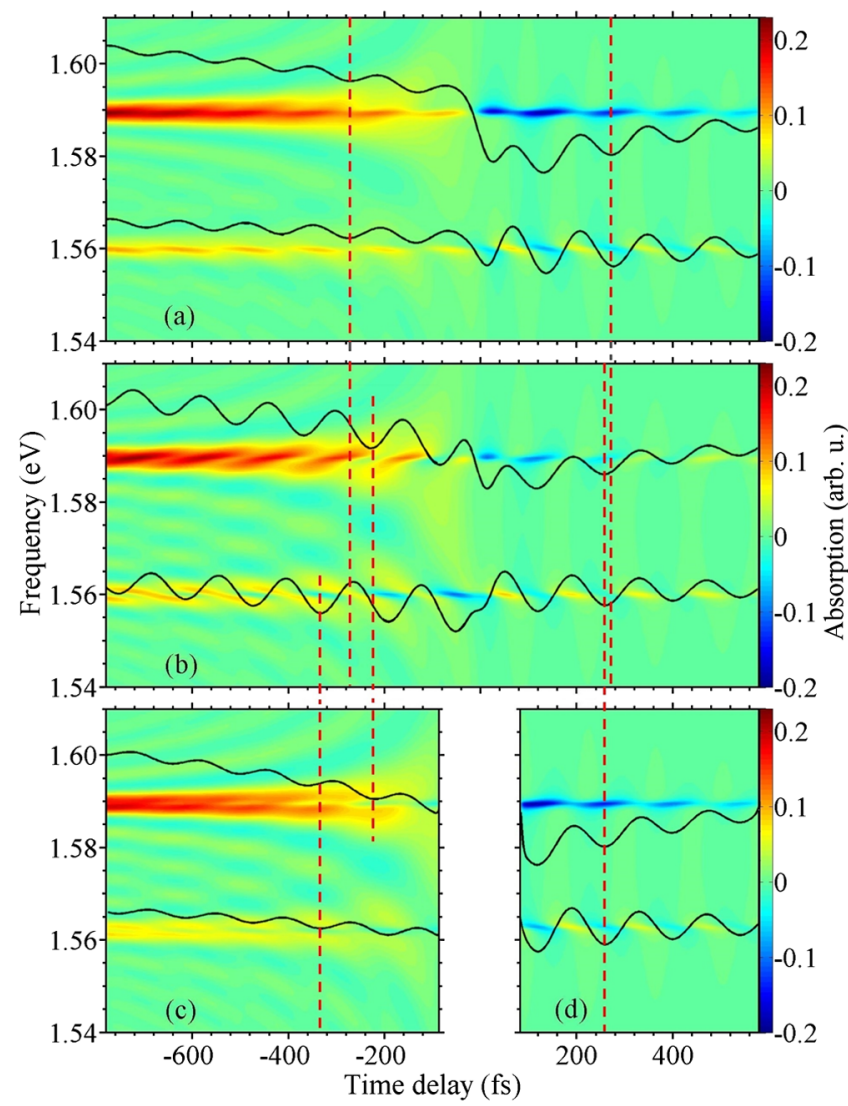

FIG. 2 (color online). Theoretical absorption spectra of a delayed probe pulse of intensity $9.2 \times 10^{8} \mathrm{~W} / \mathrm{cm}^{2}$ interacting with an ensemble of $\mathrm{Rb}$ atoms, modeled via the three-level system in Fig. 1(b) and driven at $t=0$ by a pump pulse of intensity (a) $1.0 \times 10^{10} \mathrm{~W} / \mathrm{cm}^{2}$ and (b) $2.8 \times 10^{10} \mathrm{~W} / \mathrm{cm}^{2}$. Panels (c) and (d) display, for negative and positive time delays, respectively, absorption spectra for a pump pulse of $1.0 \times$ $10^{10} \mathrm{~W} / \mathrm{cm}^{2}$ and artificial phases $\phi_{2}$ and $\phi_{3}$ [Eq. (3)], with (c) $\phi_{2}=\phi_{3}=-2.26$ for $\tau<0$, and (d) $\phi_{2}=0, \phi_{3}=0.67$ for $\tau>0$. In each panel, the top (bottom) black lines represent the absorption spectra evaluated at the transition energy $\omega_{31}\left(\omega_{21}\right)$ in arbitrary units. All black lines are on the same scale, with the 0 aligned on the corresponding transition energy.

centered on the transition energies $\omega_{21}$ and $\omega_{31}$. Furthermore, for both positive and negative time delays, amplitude and shape of the two absorption lines oscillate in $|\tau|$, with periodicity determined by the beating frequency $\omega_{32}=2 \pi /(140 \mathrm{fs})$ and a discontinuity across $\tau=0$, where the pulses overlap. The black lines highlight this behavior, displaying $\mathcal{S}\left(\omega_{21}, \tau\right)$ and $\mathcal{S}\left(\omega_{31}, \tau\right)$ evaluated at the atomic transition energies. Absorption strengths are shown in arbitrary units, as they depend on the gas pressure in the cell, which is not included in the simulation.

Figure 2(b) displays analogous features for a pump intensity of $2.8 \times 10^{10} \mathrm{~W} / \mathrm{cm}^{2}$. Here, however, different phases of the time-delay-dependent oscillations can be recognized. This is apparent from the time delays at which
$\mathcal{S}\left(\omega_{21}, \tau\right)$ and $\mathcal{S}\left(\omega_{31}, \tau\right)$ exhibit, e.g., their minima, as highlighted by the red, dashed lines. For $\tau>0$, the minima are aligned both in Figs. 2(a) and 2(b), although a common shift can be distinguished. In contrast, for $\tau<0$, the minima are aligned in Fig. 2(a), while in Fig. 2(b) they appear shifted towards opposite directions owing to the larger pump intensity. This different behavior is a consequence of the fact that, independent of the pulse order, the dipole response of the system is referenced to the arrival time $\tau$ of the detected probe pulse [Eq. (1)].

In Figs. 2(c) and 2(d) we show that the phase of the oscillations in $\tau$ encodes the key information about the intensity-dependent atomic phases. In both panels, a pump intensity of $1.0 \times 10^{10} \mathrm{~W} / \mathrm{cm}^{2}$ is used as in Fig. 2(a). Here, however, we modify the phases of the system by artificially transforming the density matrix $\hat{\rho}(T / 2, \tau)-$ describing the atomic system at $t=T / 2$ immediately after the interaction with the pump pulse-into

$$
\hat{\rho}^{\prime}(T / 2, \tau)=\hat{\Phi} \hat{\rho}(T / 2, \tau) \hat{\Phi}^{\dagger} \text {. }
$$

The usual equations of motion are employed to calculate the subsequent dynamics. In Eq. (3), $\hat{\Phi}$ is a diagonal matrix of elements $\Phi_{i j}=e^{-i \phi_{i}} \delta_{i j}$, with $\phi_{1}=0$, while $\phi_{2}$ and $\phi_{3}$ represent shifts in the phase of levels $|2\rangle$ and $|3\rangle$, respectively, such that $\rho_{i j}^{\prime}(T / 2, \tau)=\rho_{i j}(T / 2, \tau) e^{-i\left(\phi_{i}-\phi_{j}\right)}$.

These artificial shifts $\phi_{k}$ effectively model phase changes undergone by the system at varying pump intensities, to which the absorption spectra are sensitive. For instance, in Fig. 2(c), for $\tau<0$, each $\phi_{k}$ is set equal to the difference between the phase changes caused by a pump intensity of $2.8 \times 10^{10} \mathrm{~W} / \mathrm{cm}^{2}$ [Fig. 2(b)] and of $1.0 \times 10^{10} \mathrm{~W} / \mathrm{cm}^{2}$ [Fig. 2(a)], which are extracted from the corresponding dynamics of the density matrix. The resulting absorption spectrum features time-delay-dependent oscillations with the same amplitudes as in Fig. 2(a), but whose phases are now equal to those displayed in Fig. 2(b). An analogous procedure is applied in Fig. 2(d) for $\tau>0$, with new phases $\phi_{k}$.

In order to test the relation between intensity-dependent atomic phases and time-delay-dependent absorption spectra, in Fig. 3 results are shown from the experimental measurement of the OD absorption spectrum of a probe pulse transmitted through an ensemble of $\mathrm{Rb}$ atoms. A Ti: sapphire laser is utilized, with a repetition rate of $4 \mathrm{kHz}$, pulse energy of $0.7 \mathrm{~mJ}$, FWHM of $30 \mathrm{fs}$, and central wavelength of $780 \mathrm{~nm}$. The laser beam is split by a spatial mask with two irises into two beams, which are used as probe and pump beams in Fig. 1(a). A delay line is realized in the pump beam path by means of a piezo translation stage at close-to-normal incidence, allowing one to scan the time delay between the two pulses. $\mathrm{Rb}$ vapor is supplied in a cell with $20 \mathrm{~mm}$ length, which is heated to $160^{\circ} \mathrm{C}$ by a homemade heating and control system. Using the known vapor pressure of $\mathrm{Rb}$, the atomic density is estimated to be 


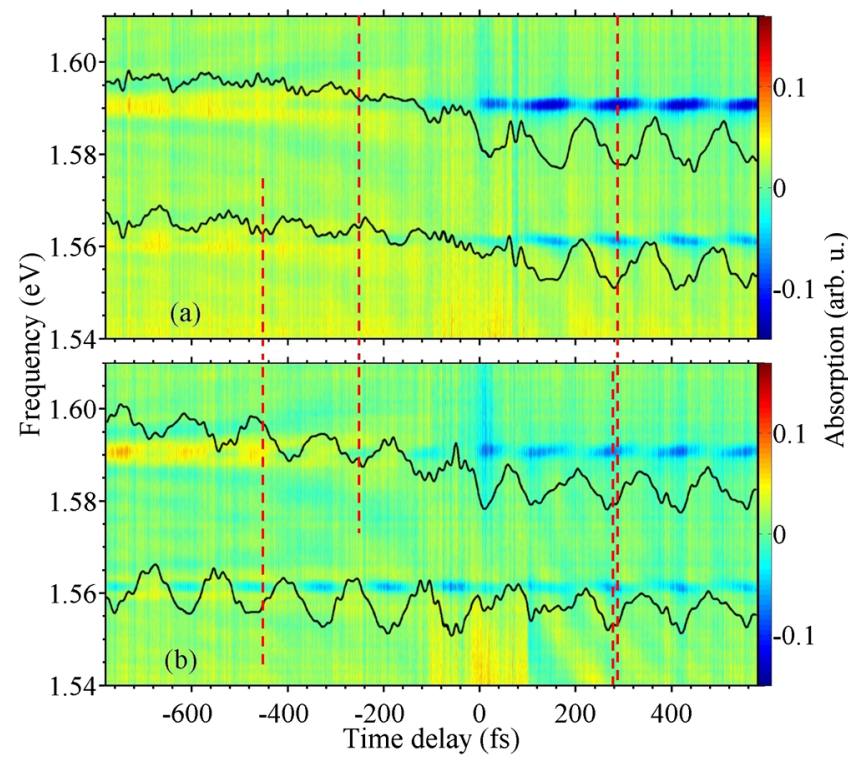

FIG. 3 (color online). Experimental absorption spectra of a delayed probe pulse of intensity $I_{\mathrm{pr}}=9.2 \times 10^{8} \mathrm{~W} / \mathrm{cm}^{2}$, transmitted through an ensemble of $\mathrm{Rb}$ atoms driven at $t=0$ by a pump pulse of intensity (a) $I_{\mathrm{pu}}=1.0 \times 10^{10} \mathrm{~W} / \mathrm{cm}^{2}$ and (b) $I_{\mathrm{pu}}=2.8 \times 10^{10} \mathrm{~W} / \mathrm{cm}^{2}$. The black on-resonance lineouts have the same meaning as in Fig. 2.

$2.8 \times 10^{14} \mathrm{~cm}^{-3}$. The pump and probe pulses, with beam sizes of $3.5 \mathrm{~mm}$ and $3.0 \mathrm{~mm}$, respectively, are focused by a concave mirror with a focusing length of $500 \mathrm{~mm}$. After passing through the sample, the probe beam is picked up by an identical concave mirror and coupled into a fiberpigtailed spectrometer (Ocean Optics, USB2000+). The probe pulse has an intensity of $9.2 \times 10^{8} \mathrm{~W} / \mathrm{cm}^{2}$ and Figs. 3(a) and 3(b) show experimental results for a pump intensity of $1.0 \times 10^{10} \mathrm{~W} / \mathrm{cm}^{2}$ and $2.8 \times 10^{10} \mathrm{~W} / \mathrm{cm}^{2}$, respectively. For low pump intensities [Fig. 3(a)], at $\tau<0$, the small amplitude of the oscillations in $\tau$ renders the associated phases not straightforwardly extractable. Apart from that, the experimental results are in good agreement with the theoretical predictions from Fig. 2, showing analogous intensity-dependent phase shifts in the oscillating features of the absorption spectra.

Finally, we focus on the dependence of phase effects upon pump-pulse intensities. Figures 4(a) and 4(b) display the experimental absorption spectra $\mathcal{S}\left(\omega_{21}, \tau\right)$ and $\mathcal{S}\left(\omega_{31}, \tau\right)$, evaluated at the transition energies $\omega_{21}$ and $\omega_{31}$, respectively, for varying pump intensities. These measurement results quantify intensity-dependent shifts in the phase of the time-delay-dependent spectra, which are in good agreement with the theoretical predictions in Figs. 4(c) and 4(d), confirming the observations from Figs. 2 and 3.

These experimentally observable phase properties reflect the different physical mechanisms acting at negative and positive time delays. This is further analyzed by implementing the artificial-phase method introduced in Eq. (3).

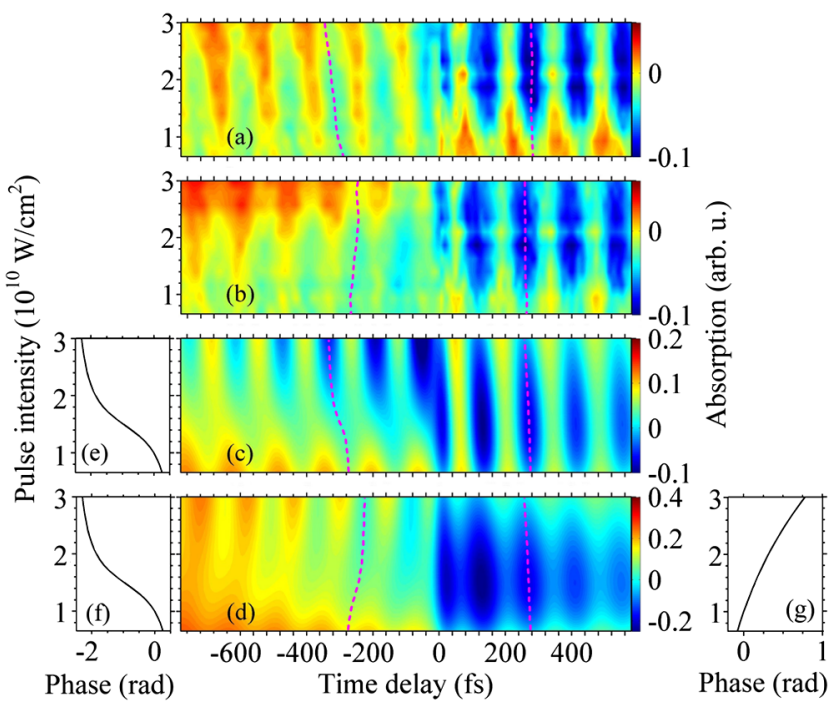

FIG. 4 (color online). [(a), (b)] Experimental and [(c), (d)] theoretical absorption spectra at fixed frequencies $\left[\omega_{21}=1.56 \mathrm{eV}\right.$ in (a) and (c), $\omega_{31}=1.59 \mathrm{eV}$ in (b) and (d)] of a delayed probe pulse of intensity $I_{\mathrm{pr}}=9.2 \times 10^{8} \mathrm{~W} / \mathrm{cm}^{2}$, transmitted through an ensemble of $\mathrm{Rb}$ atoms driven at $t=0$ by a pump pulse of varying intensity. The dashed, magenta lines display the time delays (as a function of pump intensity) associated with minima in the absorption spectra. Panels (e), (f), and (g) show the artificial phases $\left[\phi_{2}\right.$ in (e), $\phi_{3}$ in (f) and (g), see also Eq. (3)] which need be added at $I_{\mathrm{pu}}=1.0 \times 10^{10} \mathrm{~W} / \mathrm{cm}^{2}$ to reproduce the intensitydependent phases of the time-delay-dependent oscillations at [(e), (f)] negative and $(\mathrm{g})$ positive time delays.

For $\tau<0$, the pump pulse strongly modifies the excited state generated by the probe pulse. The intensity-dependent phases of the oscillations in $\tau$ featured in Figs. 4(c) and 4(d) enable one to quantify the shifts in the phase of the atomic coherences ensuing from the interaction with strong pump pulses of varying intensity, as captured by $\phi_{2}\left(I_{\mathrm{pu}}\right)$ and $\phi_{3}\left(I_{\mathrm{pu}}\right)$ in Figs. 4(e) and 4(f), respectively. In contrast, for $\tau>0$, the pump pulse is responsible for the initial excitation of the system and the generation of an initial phase difference $\Delta \phi$. The parallel shift undergone by $\mathcal{S}\left(\omega_{21}, \tau\right)$ and $\mathcal{S}\left(\omega_{31}, \tau\right)$ at increasing pump intensities [Figs. 4(c) and 4(d)] is a consequence of the phase $\omega_{32} \Delta \tau$ necessary to compensate for $\Delta \phi$. Artificial phases are used to effectively reproduce this behavior, confirming that the phases of the resulting spectra $\mathcal{S}\left(\omega_{21}, \tau\right)$ and $\mathcal{S}\left(\omega_{31}, \tau\right)$ are identically affected only by the intensitydependent phase difference $\phi_{3}\left(I_{\mathrm{pu}}\right)-\phi_{2}\left(I_{\mathrm{pu}}\right)$. This is shown in Fig. $4(\mathrm{~g})$ for $\phi_{2}\left(I_{\mathrm{pu}}\right)=0$. To include the effect of experimental spatial pulse profiles in our calculations, the intensity-dependent spectra would have to be averaged over the intensity distribution in the experimental interaction volume. The slow monotonic shape of the intensitydependent phases exhibited in Fig. 4, however, suggests that intensity averaging would not essentially modify the phase effects investigated in this work, yielding the good agreement between theory and experiment in Fig. 4. 
In conclusion, we investigated and interpreted timedelay-dependent absorption spectra and their relationship to the phase evolution and intensity dependence of atomic quantum coherences. An artificial-phase method was introduced to analyze intensity-dependent phase effects, but the level-specific phases $\phi_{k}$ could also model additional interactions with external fields selectively modifying the two excited states. Understanding the phase-sensitive link between quantum dynamics and transient-absorption spectra represents an essential step towards the application of TAS to the extraction of quantum phases in bound-state atomic systems interacting with strong-field pulses, thus allowing for more sensitive tests of quantum-dynamics theory.

The authors acknowledge support from the Max-Planck Research Group program, the DFG (Grant No. PF790/301 1-1), and the ERC (Grant No. X-MuSiC-616783). Z. L. acknowledges the scholarship award for excellent doctoral students granted by the Ministry of Education of China. Z. L., S. M. C., and T. P. conceived and developed the key idea; Z. L., C. O., K. M., and Y. M. conducted the experiments; S.M.C. developed the theoretical model and performed the numerical calculations; the paper was written by S. M. C. (theory and interpretation) and Z. L. (experiment), with contributions from all coauthors.

Z. L. and S. M. C. contributed equally to this work.

*liuzy04@yeah.net

†smcavaletto@gmail.com

tpfeifer@mpi-hd.mpg.de

[1] T. Brabec and F. Krausz, Intense few-cycle laser fields: Frontiers of nonlinear optics, Rev. Mod. Phys. 72, 545 (2000).

[2] F. Krausz and M. Ivanov, Attosecond physics, Rev. Mod. Phys. 81, 163 (2009).

[3] S. R. Leone, C. W. McCurdy, J. Burgdörfer, L.S. Cederbaum, Z. Chang, N. Dudovich, J. Feist, C. H. Greene, M. Ivanov, R. Kienberger, U. Keller, M. F. Kling, Z.-H. Loh, T. Pfeifer, A. N. Pfeiffer, R. Santra, K. Schafer, A. Stolow, U. Thumm, and M. J. J. Vrakking, What will it take to observe processes in 'real time'?, Nat. Photonics 8, 162 (2014).

[4] R. A. Mathies, C. H. Brito Cruz, W. T. Pollard, and C. V. Shank, Direct observation of the femtosecond excited-state cis-trans isomerization in bacteriorhodopsin, Science $\mathbf{2 4 0}$, 777 (1988).

[5] W. T. Pollard and R. A. Mathies, Analysis of femtosecond dynamic absorption spectra of nonstationary states, Annu. Rev. Phys. Chem. 43, 497 (1992).

[6] Z.-H. Loh, M. Khalil, R. E. Correa, R. Santra, C. Buth, and S.R. Leone, Quantum State-Resolved Probing of Strong-Field-Ionized Xenon Atoms Using Femtosecond High-Order Harmonic Transient Absorption Spectroscopy, Phys. Rev. Lett. 98, 143601 (2007).

[7] H. Wang, M. Chini, S. Chen, C.-H. Zhang, F. He, Y. Cheng, Y. Wu, U. Thumm, and Z. Chang, Attosecond
Time-Resolved Autoionization of Argon, Phys. Rev. Lett. 105, 143002 (2010).

[8] E. Goulielmakis, Z.-H. Loh, A. Wirth, R. Santra, N. Rohringer, V. S. Yakovlev, S. Zherebtsov, T. Pfeifer, A. M. Azzeer, M. F. Kling, S. R. Leone, and F. Krausz, Real-time observation of valence electron motion, Nature (London) 466, 739 (2010).

[9] M. Holler, F. Schapper, L. Gallmann, and U. Keller, Attosecond Electron Wave-Packet Interference Observed by Transient Absorption, Phys. Rev. Lett. 106, 123601 (2011).

[10] U. Fano and J. W. Cooper, Spectral distribution of atomic oscillator strengths, Rev. Mod. Phys. 40, 441 (1968).

[11] R. Kienberger, E. Goulielmakis, M. Uiberacker, A. Baltuska, V. Yakovlev, F. Bammer, A. Scrinzi, Th. Westerwalbesloh, U. Kleineberg, U. Heinzmann, M. Drescher, and F. Krausz, Atomic transient recorder, Nature (London) 427, 817 (2004).

[12] J. Mauritsson, T. Remetter, M. Swoboda, K. Klünder, A. L'Huillier, K. J. Schafer, O. Ghafur, F. Kelkensberg, W. Siu, P. Johnsson, M. J. J. Vrakking, I. Znakovskaya, T. Uphues, S. Zherebtsov, M. F. Kling, F. Lépine, E. Benedetti, F. Ferrari, G. Sansone, and M. Nisoli, Attosecond Electron Spectroscopy Using a Novel Interferometric Pump-Probe Technique, Phys. Rev. Lett. 105, 053001 (2010).

[13] S. Chen, M. J. Bell, A. R. Beck, H. Mashiko, M. Wu, A. N. Pfeiffer, M. B. Gaarde, D. M. Neumark, S. R. Leone, and K. J. Schafer, Light-induced states in attosecond transient absorption spectra of laser-dressed helium, Phys. Rev. A 86, 063408 (2012).

[14] A. Kaldun, C. Ott, A. Blättermann, M. Laux, K. Meyer, T. Ding, A. Fischer, and T. Pfeifer, Extracting Phase and Amplitude Modifications of Laser-Coupled Fano Resonances, Phys. Rev. Lett. 112, 103001 (2014).

[15] U. Fano, Effects of configuration interaction on intensities and phase shifts, Phys. Rev. 124, 1866 (1961).

[16] C. Ott, A. Kaldun, P. Raith, K. Meyer, M. Laux, J. Evers, C. H. Keitel, C. H. Greene, and T. Pfeifer, Lorentz meets Fano in spectral line shapes: A universal phase and its laser control, Science 340, 716 (2013).

[17] K. P. Heeg, C. Ott, D. Schumacher, H.-C. Wille, R. Röhlsberger, T. Pfeifer, and J. Evers, Interferometric Phase Detection at X-Ray Energies via Fano Resonance Control, Phys. Rev. Lett. 114, 207401 (2015).

[18] S. M. Cavaletto, Z. Harman, C. Ott, C. Buth, T. Pfeifer, and C. H. Keitel, Broadband high-resolution X-ray frequency combs, Nat. Photonics 8, 520 (2014).

[19] Z. Liu, C. Ott, S. M. Cavaletto, Z. Harman, C. H. Keitel, and T. Pfeifer, Generation of high-frequency combs locked to atomic resonances by quantum phase modulation, New J. Phys. 16, 093005 (2014).

[20] R. Netz, T. Feurer, G. Roberts, and R. Sauerbrey, Coherent population dynamics of a three-level atom in spacetime, Phys. Rev. A 65, 043406 (2002).

[21] M. O. Scully and M.S. Zubairy, Quantum Optics (Cambridge University Press, Cambridge, England, 1997).

[22] C. J. Foot, Atomic Physics (Oxford University Press, Oxford, 2005).

[23] W. R. Johnson, Atomic Structure Theory: Lectures on Atomic Physics (Springer, Berlin Heidelberg, New York, 2007). 